\title{
DEPENDENCE OF WORK FUNCTION ON SURFACE MAGNETIZATION*
}

\author{
J. BACZYŃSKI AND L. WOJTCZAK \\ Institute of Physics, University of Eódź \\ Pomorska 149/153, 90-236 Łódź, Poland
}

(Received July 29, 1991)

\begin{abstract}
It is shown theoretically that, in contradiction to massive specimens, the work function of ferromagnetic thin films does not depend linearly on the square of magnetization as a consequence of the inhomogeneous magnetization distribution. This theoretical conclusion is confirmed by experimental investigations of gadolinium films as well as interpretation of the results reported for nickel samples.
\end{abstract}

PACS numbers: 75.70. $-\mathrm{i}, 73.30 .+\mathrm{y}$

\section{Introduction}

The distribution of the magnetization across a sample in the form of a thin film has been a subject of experimental investigations connected with the Mössbauer spectroscopy [1]. The evidence of the increase of magnetization at the surface in low temperatures has been recently confirmed by the same Mössbauer technique for very thin iron films [2].

The effects reflecting the existence of magnetization inhomogeneity at the surface are intensively considered. One of them seems to be the dependence of the work function on the magnetization of a sample and its surface inhomogeneity particularly in the interval close to the Curie temperature. The dependence was reported by Vonsovsky and Sokolov [3] who predicted the linear relation between the work function $W$ and the square of the homogeneous magnetization value $M$. The influence of the spontaneous magnetization on the work function was also confirmed by Denisov et al. [4] in their experiments on nickel samples in which the spontaneous magnetization was treated as homogeneous. However, these experimental results show that there is a deviation of the linear relation of $W$ with respect to $M^{2}$. We can see on Fig. 1 that the experimental points are better related

\footnotetext{
*This work was supported by the Project CPBP 01.08.
} 


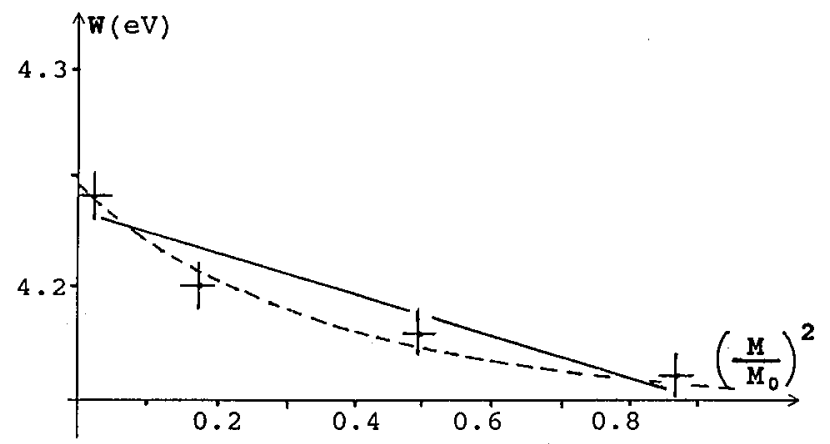

Fig. 1. The experimental dependence of the work function $W$ on the magnetization $M$ for a nickel sample [4]. $M_{0}=M\left(T=T_{\mathrm{C}}\right), T_{\mathrm{C}}-$ Curie temperature. - the curve given in [4] according to the theory of Vonsovsky, - - - the curve fitted according to the formula (5).

to the curve indicated by the dashed line than to a straight line representing the linear relation suggested in [4]. This remark has been a starting point of our considerations connected with the work function of gadolinium and its relation to the magnetization dependence. An analysis of the results obtained by the vibrating capacitor method [5] has shown that the form of the relation for $W$ proportional to the term of the square of magnetization and including the linear term with respect to the magnetization describes the experimental situation better than the relation previously mentioned. In order to proceed to the experimental procedure we begin with the theoretical discussion of the influence of magnetization inhomogeneities on the behaviour of the work function of ferromagnetic materials. The first part refers to the theoretical considerations. It is evident that the spontaneous magnetization is inhomogeneous mainly at the surfaces, and the magnetization distribution is expected to be inhomogeneous across the film thickness. It seems to us therefore to be natural to analyse the work function dependence with respect to the local magnetization in the case of thin films which are the most suitable object for the discussion of the surface properties, when the samples are sufficiently thick (i.e. the film thickness $n$ is tending to infinity).

The second part of the paper is devoted to the experimental technique adapted to the evaluation of the function $W$ with respect to $M$. The testing measurements are made in the case of gadolinium samples which are very convenient for the study because of the low value of the Curie temperature as well as a sufficiently large magnetic moment. The experimental method is based on the measurements of the contact potentials by means of the vibrating capacitor technique.

The third part brings interpretation of experimental results and suitable analysis for a gadolinium sample as a very useful method proposed for qualitative detection of the decrease or increase of the magnetization at the surface with respect to its a verage value. 


\section{Theoretical considerations: spontaneous magnetization influence on work function}

The work function can be measured in experiments as a quantity characteristic of a given sample. From the theoretical point of view it is determined by the properties of the band structure of the considered system and therefore it should be connected with the surface states because of their fundamental influence on the local density of electrons.

Next, we would like to remark that the work function is determined by the Fermi level including the surface barrier. Thus the density of states is a basic information for our considerations. Of course, the density of states depends on the distribution of electron occupation numbers with respect to their spins. It means that the work function depends on the spontaneous magnetization below Curie temperature [3]. Additionally, it is well-known from the thin film theory (e.g. [6]) that the spontaneous magnetization is not homogeneous in the surface region. It is also evident that the distribution of the spontaneous magnetization across the film thickness leads to the deviation from the linear relation between the work function and the square of the average magnetization value in the one-band model [7].

The influence of the boundary condition on the work function behaviour was studied in connection with the interface properties from the point of view of electrochemistry [8]. It turns out that it is necessary to include the surface potential barrier which contributes to the Fermi level as a local quantity with respect to monoatomic layers parallel to the surface.

The one-band model describes the situation when the electron emission is considered from the band responsible for magnetic order. In fact, the process is more complicated from the physical point of view. Namely, the electrons are emitted from the conducting band (i.e. $s$-band) while the spontaneous magnetization results from the magnetic band (i.e. $d$-band or $f$-band). Therefore the model describing the dependence of the work function on the magnetization should take into account at least two coupled bands. The procedure concerning the coupling between magnetic and conducting bands is reported in [9]. The present paper brings an application of the theory to the case of thin films where the surface states play an essential role.

Now we present the outline of the considerations leading to the description of the influence of the spontaneous magnetization inhomogeneity on the work function when the boundary conditions are taken into account.

The work function $W$ is defined as the energy which is necessary to carry out an electron from the Fermi level at the surface of a sample. It means that the work function can be measured by the difference between the energy $W_{\mathbf{B}}$ of the last filled band bottom and the Fermi level at the surface calculated with respect to the band bottom energy, namely (see Fig. 2):

$$
W=-W_{\mathrm{B}}(\nu=1)+E_{\mathrm{F}} \quad \text { or } \quad W=-W_{\mathrm{B}}+E_{\mathrm{F}}-\Delta \Phi .
$$

Hence, we can write [8]:

$$
W=-W_{\mathrm{B}}+E_{\mathrm{F}}+I\left(\bar{n}_{\mathrm{e}}-n_{1}\right),
$$



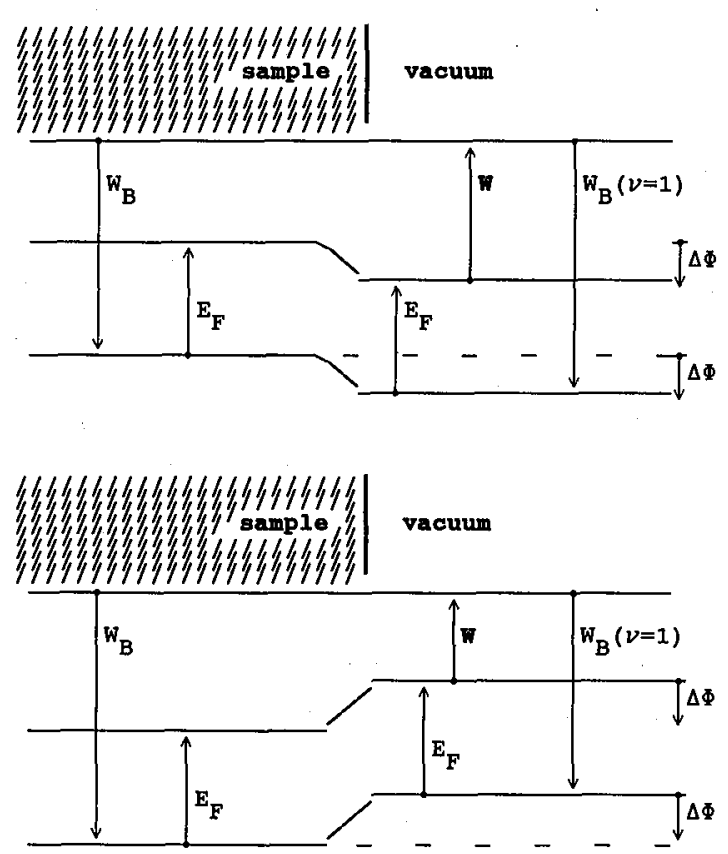

Fig. 2. The schematic energy level diagram.

where the monoatomic layers parallel to the surface are labelled by $\nu \in(1, n)$ and $n$ is the number of layers corresponding to the thickness of a sample, the parameter $I$ denotes the Coulomb integral for the conducting band, $n_{\nu}$ is the number of electrons in the layer $\nu$ (i.e. $n_{1}$ is the number of electrons at the surface) while $\bar{n}_{\mathrm{e}}$ means the average value of the electrons in the considered band.

The calculation of the Fermi level appearing in thin films as reported in [10] are based on the distribution of electrons expressed in terms of the averaged values $\nu, m, \sigma$ for the electron number operators belonging to the considered band $m$ with the spin orientation $\sigma$ in a given layer. Then the Fermi level $E_{\mathrm{F}}$ is determined by the condition:

$$
\sum_{\nu m \sigma}\left\langle n_{\nu m \sigma}\right\rangle=\tilde{n}_{\mathrm{e}} n
$$

when $\tilde{n}_{\mathrm{e}}$ stands for the average number of electrons per atom. When the calculations of the Fermi level refer to the conducting valence band then the sum over $m$ is taken only for $m$ corresponding to the considered band.

In the case of the samples restricted by the surface but sufficiently thick the Fermi level determined by (2) can be calculated by means of the distributions of electrons for the homogeneous system whose magnetization is described by its average value.

The surface effects are connected with the term $I\left(\tilde{n}_{\mathrm{e}}-n_{1}\right)$ only. Taking into account the usually accepted simplified assumptions [e.g. 10] that the distribution 
of electrons is of the form $\left\langle n_{1 \uparrow}\right\rangle=(1+\chi)\left\langle n_{\uparrow}\right\rangle,\left\langle n_{1 \downarrow}\right\rangle=(1-\chi)\left\langle n_{\downarrow}\right\rangle$ we obtain:

$$
I\left(n_{\mathrm{e}}-n_{1}\right)=-2 I \chi \sigma,
$$

where $\sigma=\frac{1}{2}\left(n_{\uparrow}-n_{\downarrow}\right)$ denotes the average magnetization of the conducting band and $\chi$ stands for the surface magnetization parameter $\sigma_{1}$, i.e. $\sigma_{1}=\sigma\left(1+\chi \tilde{n}_{\mathrm{e}} / 2 \sigma\right)$.

The properties of the magnetization parameter $\sigma$ as well as the distribution of electrons $\left\langle n_{\nu s \sigma}\right\rangle$ are determined by the coupling between the conducting and magnetic bands. The theory of the interacting band model [9] for a homogeneous system shows that we can write $\sigma=\gamma M$ where $M$ is the spontaneous magnetization of the $d$ - (or $f$-) bands and $\gamma$ is the parameter calculated from the hybrydization equations [9]. Finally we obtain:

$$
E_{\mathrm{F}}(M)=\frac{E_{\mathrm{F}}}{2}\left[1+\sqrt{1-\kappa^{2}\left(\frac{M}{M_{0}}\right)^{2}}\right],
$$

where

$$
E_{\mathrm{F}}=t a^{2}\left(3 \pi \tilde{n}_{\mathrm{e}}\right)^{\frac{2}{3}} \quad \text { and } \quad \kappa=2 S \frac{I \gamma+B}{E_{\mathrm{F}}}
$$

are determined by the model parameters. It is not necessary to know these parameters for a simple analysis of the surface magnetization because the relations between them and coefficients estimated directly from the experiment allow us to determine the sign of $\chi$.

Substituting (3) and (4) into (1) we can see that the work function is described by two terms; $E_{\mathrm{F}}$ corresponding to the homogeneous system (volume effects) and $2 I \chi \sigma$ reflecting a contribution given by the inhomogeneous part of the system (surface effects).

In consequence we can see that the work function depends on the square of magnetization when the system is magnetically homogeneous and the linear term with respect to the magnetization appears only for the distribution of magnetization at the surface.

\section{Experimental technique}

In order to test our theoretical predictions for various experimental situations the experimental technique for the measurements of the function $W=W(M)$ has been arranged. For this purpose the method for simultaneous measurement of the contact potential difference (CPD) and the point of zero charge (PZC) in electrolytic solution [11] has been applied. The work function can be observed by direct measurement of CPD as a function of temperature. The method is then especially convenient for the investigations of heterogeneous surfaces at which various points have very different values of $W$. It gives the arithmetic mean work function of a heterogeneous surface, whereas direct methods give an average that is weighted in favour of low work function values. Unlike many other methods, the calculation of the work function does not depend on an estimation of the 
electron reflection coefficient of the surface. It does not use high temperature, high electric fields, or beams of electrons or photons, and thus it does not run the risk of moving the adsorbate to new sites on the surface, nor of desorbing it [5]. The CPD method is a particular method of measuring the work function. It can be used without any a priori predictions of the density states, contrary to other methods, e.g. Fowler's method. The present technique allows us to compare experimental results of various methods because of their independence of the systematic errors of experiment and simultaneously it allows us to discuss the theoretical predictions without the influence of the applied technique. From the experimental point of view we can prove it by heating the specimen to temperatures higher than the Curie point. In the paramagnetic phase the temperature dependence of the work function is stabilized, while below $T_{\mathrm{C}}$ we can observe this dependence being in agreement with the theoretical prediction.

The changes of the value $W$ are observed by the CPD measurements between the reference surface and the investigated one. Thus $W=W_{\mathrm{Ref}}+e U_{v}$, where $U_{v}$ stands for CPD and $e$ is the electron charge. The measurements of $U_{v}$ were made by means of the vibrating capacitor method. The equipment was described in Ref. [12]. The experimental error of the CPD values in the present paper is $\pm 5 \mathrm{mV}$. In fact, in the experiment for a gadolinium specimen we obtained the relation $\Delta W=W\left(T=T_{\mathrm{C}}\right)-W(T)$. In order to compare the dependence $W=W(M)$ with the theoretical predictions we should rewrite the magnetization by its temperature dependence. We use consequently the expression for the magnetization predicted by the Green function theory which is applied in order to obtain the formula for the work function. In the neighbourhood of the Curie temperature we have $\left(M / M_{0}\right)^{2}=\left(T_{\mathrm{C}}-T\right) / T_{\mathrm{C}}$. Then taking into account the formula (1) we can see that

$$
W=W_{0}+\Delta W=W_{0}+\lambda_{1}\left(\frac{M}{M_{0}}\right)-\lambda_{2}\left(\frac{M}{M_{0}}\right)^{2},
$$

where the parameters $\lambda_{1}$ and $\lambda_{2}$ are fitted to the experimental data and they are related to the model parameters.

The formula (1) for the expressions (3) and (4) can be considered in the form (5) with the relations between the parameters of the theory, $\lambda_{1}=-2 I \gamma\langle S\rangle\langle\chi\rangle$, $\lambda_{2}=(I \gamma S+B S)^{2} E_{\mathrm{F}}^{-1}$, where $E_{\mathrm{F}}=E_{\mathrm{F}}(M=0)$, hence the parameter

$$
\chi=\frac{\lambda_{1}}{2}\left(B S-\sqrt{\lambda_{2} E_{\mathrm{F}}}\right)^{-1}
$$

indicates qualitatively that the magnetization inhomogeneities appear at the surface and it determines the lowering or the increasing of the magnetization at the surface with respect to the mean value of magnetization. It is worthwhile to notice that $\lambda_{2}$ should be always positive and the sign of $\chi$ depends on the product of the sign $\lambda_{1}$ and the sign of $\left(\sqrt{\lambda_{2} E_{\mathrm{F}}}-B S\right)$, i.e. the signs of the model parameters characterizing the material.

According to the introduced assumptions we obtain:

$$
\frac{\Delta M}{M}=\frac{\chi \tilde{n}_{\mathrm{e}}}{2 \sigma}
$$


for $\Delta M=M_{1}-M$ so that the parameter $\chi$ shows the lowest deformation for the maximal value of $\sigma$ (e.g. $\sigma=1 / 2$ and $\tilde{n}_{\mathrm{e}}=1$ ). In this case $(\Delta M / M)=\chi$.

\section{Interpretation: gadolinium samples}

We apply the considerations presented in the paragraph 2 to the case of the band model adapted to gadolinium samples [13]. First of all, we report some information concerning gadolinium properties. Namely, the gadolinium monocrystals have the HCP crystallographic structure with the lattice constants $a=3.6360 \AA$ and $c=5.7826 \AA$ [14]. The Curie temperature is $T_{\mathrm{C}}=290.85 \mathrm{~K}$ and the saturation magnetization $\mu=2010 \mathrm{Gs} \mathrm{cm}^{-2}$ at $T=0 \mathrm{~K}$ [15]. The electronic structure shows that the highest occupied level $6 s$ with the energy $E_{6 s}=-0.3878 \mathrm{Ry}$ [16] (in an atom) is completely filled while the levels $4 f$ and $5 d$ are not completely filled and they are occupied by electrons with the same orientation of their spins. The energies of electronic levels in an isolated atom are $E_{5 d}=-0.4363$ Ry [16] and $E_{4 f}=-1.5912 \mathrm{Ry}$ [16]. The next level $5 p$ has energy $E_{5 p}=-2.2693$ Ry [16], i.e. it is sufficiently far from uncompleted occupied levels and can be treated as belonging to an electronic cloud of the hard-core potential. The electronic struc-

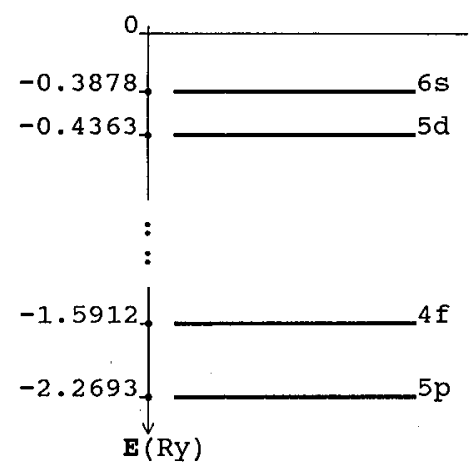

Fig. 3. The energy levels in the gadolinium atom.

ture is shown schematically on Fig. 3 where the numbers of electrons $\tilde{n}_{d} \approx 1$ and $\tilde{n}_{f} \approx 7.1$ correspond to the spin value $S_{d}=\frac{1}{2}$ and $S_{f}=\frac{7}{2}$.

Taking into account the above properties of the electronic structure of gadolinium we can consider the band model for three or at least two effective bands which are responsible for the electronic emission (band $6 s$ ) and for the spontaneous magnetization (bands $5 d$ and $4 f$ ). The effective magnetic band can be considered as the coupled bands with localized electrons whose spins are oriented in the same direction with the effective value $S=4$. The coupling between magnetic and the conducting bands is given by the parameter $B$, while $\sigma=\gamma M$ with $M$ determined by the function approximated by the temperature dependence equivalent to the molecular field estimations. The bottom of the energy scale should be chosen for 
$E_{4 f}$ although this fact is not essential because the experimental precision concerns only the differences between measurements in two temperature points.

The description of the work function in the gadolinium sample can be related to the formula (5) with the parameters $\lambda_{1}$ and $\lambda_{2}$ referred to the spontaneous magnetization inhomogeneity parameter $\chi$ introduced as the ratio of the surface magnetization deviation $\Delta M$ with respect to the magnetization value $M$ of a sample (c.f. [7]). The experimental dependence $\Delta W$ on the expression $\left(M / M_{0}\right)^{2}$ is displayed on the Fig. 4 . The value $W_{0}=W\left(T=T_{\mathrm{C}}\right)$ was determined by the

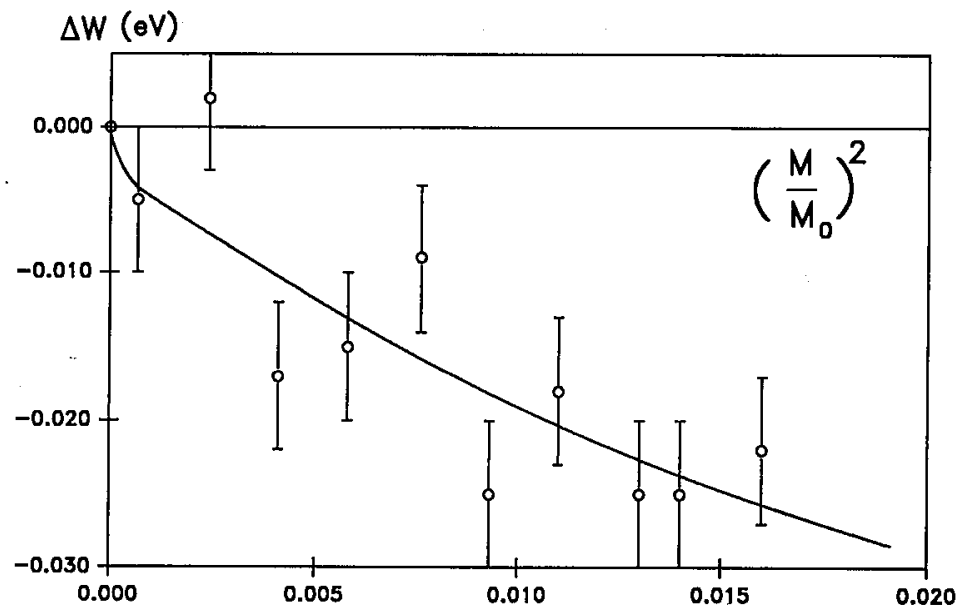

Fig. 4. The experimental dependence of the work function changes $\Delta W$ on the magnetization $M$ for a gadolinium sample. $M_{0}=M\left(T=T_{\mathrm{C}}\right), T_{\mathrm{C}}-$ Curie temperature.

photoelectric technique and it was equal to $W_{0}=3.55 \pm 0.15 \mathrm{eV}$ [11].

The best fit to the experimental data for the gadolinium sample in the present paper leads to the results: $\lambda_{1}=-0.628$ and $\lambda_{2}=+0.124$.

Taking into consideration the estimation of the parameters $\lambda_{1}$ and $\lambda_{2}$ we see that $\lambda_{2}$ confirms its sign, $\lambda_{1}$ shows an increase of magnetization $M$ at the surface when $\sqrt{\lambda_{2} E_{\mathrm{F}}}>B S$ and a decrease of $M$ when $\sqrt{\lambda_{2} E_{\mathrm{F}}}<B S$. The expected lowering of the magnetization at the surface for $T \rightarrow T_{\mathrm{C}}$ is consistent with the large value of $B(S=4)$ which assumes the maximal polarization $\sigma=1 / 2$.

\section{Interpretation: nickel samples}

In the light of the consideration reported below we can interpret the experimental data obtained by Denisov et al. [4] in terms of the deviations of $W(M)$ from the linear relation $W=W\left(M^{2}\right)$. On the basis of their experimental results we can see that the deviations from the function

$$
W=W_{0}\left(1-\lambda M^{2} / M_{0}^{2}\right)
$$


are sufficiently large and first of all bigger than the experimental error for the reported data which are collected by means of the photoemission technique (see Fig. 1). The deviations are more significant in the case when a sample is granular than in the case of continuous material (c.f. [4]). This fact qualitatively confirms the theoretical considerations because the granular sample surface is much larger than the surface of a bulk specimen.

Our proposed fit of type (5) for the data taken from the original figure [4] gave us $\lambda_{1}=-0.08$ and $\lambda_{2}=0.025$. The obtained dependence $W(M)$ is displayed on Fig. 1 as the dashed line. We can see that the magnetization decreases at the surface for sufficiently strong interband interaction.

\section{Final remarks}

The considerations presented here show that the distribution of magnetization at the surface influences the behaviour of the work function and the inhomogeneity of magnetization can be expressed by means of the deviation of the contact potential measured in a function of temperature from its linear shape with respect to the square of temperature. The degree of inhomogeneity is characterized by the surface magnetization to the average magnetization across a sample. The estimations were made for gadolinium as well as for nickel samples.

In the light of theoretical interpretation confirmed by the measurements reported in this paper it seems to us that the presented results bring a new possibility of an experimental technique for the investigations of the magnetization distribution. This method is very useful for qualitative, simple detection of the decrease or increase of the magnetization at the surface with respect to its average value. Of course, the method allows us to consider the behaviour of magnetization distribution in a more qualitative way but in this case it requires some additional theoretical relations between the band model parameters.

\section{References}

[1] S. Duncan, A. Owens, J.C. Walker, in ICMFS9 Booklet (International Colloquium on Magnetic Films and Surfaces 9), University of Eódź, Lódź 1979, p. 279.

[2] J. Korecki, in Proceeding of Conference on Surface Physics, Institute of Physics Vol. II, Eódź 1986, p. 22.

[3] S.V. Vonsovsky, A.W. Sokolov, Dokl. Akad. Nauk SSSR 76, 197 (1951).

[4] G.N. Denisov, S.W. Temko, W.D. Jagodovski, Fiz. Met. Metalloved. 39, 4 (1975).

[5] N.A. Surplice, R.J. D'Arcy, J. Phys. E, Sci. Instrum. 3, 477 (1970).

[6] L. Valenta, L. Wojtczak, J. Phys. B-30, 1025 (1980).

[7] K. Glowacki, L. Wojtczak, Report for the MR I-9 Project -1977, also presented at the Seminar of the Contemporary Problems in Quantum Chemistry, Rychtrovy Boudy 1978, (unpublished).

[8] S. Romanowski, W. Stasiak, L. Wojtczak, Electrochimica Acta 27, 511 (1982). 
[9] L. Wojtczak, J. Baczyński (unpublished results).

[10] S. Szczeniowski, L. Wojtczak, A. Sukiennicki, J. Vac. Sci. Technol. 10, 693 (1973).

[11] J. Baczyński, Ph. D. Thesis, University of Eódź, 1981.

[12] J. Baczyński, H. Małecki, M. Stępiński, Prib. Tekh. Eksp. 4, 205 (1979).

[13] J. Baczyński, S. Michalak, L. Wojtczak, XXVII Zjazd Fizyków Polskich, Lublin 1981, p. 4 (in Polish).

[14] W.B. Pearson, Lattice Spacing and Structure of Metals and Alloys, Pergamon Press, London 1958.

[15] C. Kittel, Introduction to Solid State Physics, Wiley, ed. 3, New York 1966.

[16] F. Herman, S. Skillman, Atomic Structure Calculations, Prentice Hall Inc., Englewood Cliffs, N. J. 1963. 